\title{
On the entrainment coefficient in a forced plume: quantitative effects of source parameters
}

\author{
A. Matulka ${ }^{1}$, P. López ${ }^{2}$, J. M. Redondo ${ }^{1}$, and A. Tarquis ${ }^{3}$ \\ ${ }^{1}$ Department of Applied Physics, UPC, Barcelona, Spain \\ ${ }^{2}$ Department of Applied Mathematics, UCM, Madrid, Spain \\ ${ }^{3}$ Department of Applied Mathematics, UPM, Madrid, Spain
}

Correspondence to: P. López (maplopez@bio.ucm.es)

Received: 31 May 2013 - Revised: 20 December 2013 - Accepted: 7 January 2014 - Published: 24 February 2014

\begin{abstract}
The behavior of a forced plume is mainly controlled by the source buoyancy and momentum fluxes and the efficiency of turbulent mixing between the plume and the ambient fluid (stratified or not). The interaction between the plume and the ambient fluid controls the plume dynamics and is usually represented by the entrainment coefficient $\alpha_{\mathrm{E}}$. Commonly used one-dimensional models incorporating a constant entrainment coefficient are fundamental and very useful for predictions in geophysical flows and industrial situations. Nevertheless, if the basic geometry of the flow changes, or the type of source or the environmental fluid conditions (e.g., level of turbulence, shear, ambient stratification, presence of internal waves), new models allowing for variable entrainment are necessary. The presented paper is an experimental study based on a set of turbulent plume experiments in a calm unstratified ambient fluid under different source conditions (represented by different buoyancy and momentum fluxes). The main result is that the entrainment coefficient is not a constant and clearly varies in time within the same plume independently of the buoyancy and the source position. This paper also analyzes the influence of the source conditions on the mentioned time evolution. The measured entrainment coefficient $\alpha_{\mathrm{E}}$ has considerable variability. It ranges between 0.26 and 0.9 for variable Atwood number experiments and between 0.16 and 0.55 for variable source position experiments. As is observed, values are greater than the traditional standard value of Morton et al. (1956) for plumes and jets, which is about 0.13.
\end{abstract}

\section{Introduction}

Forced plumes play a fundamental role in a large variety of natural phenomena and industrial processes. Understanding the dynamics of plumes issuing from industrial chimneys or those generated by forest fires or volcanoes is a major goal for environmental sciences because they are able to transport toxic gas and fine particles into the high atmosphere. River plumes are another natural plume phenomenon. These are turbid freshwaters flowing from land and generally in the distal part of a river outside the bounds of an estuary or river channel. Submarine plumes are another example of natural flows. The hot fluid rises into the cold ocean (Carazzo et al., 2008). Plumes, jets, and thermals are important concepts (Squires and Turner, 1962) for describing particular cases of atmospheric convection. Looking at a large thunderstorm, an analogy with a steady-state buoyant turbulent plume can be suggested, having a continuous source of heat from below the cloud base. On the other hand, for small clouds, being about as deep as wide, a non-steady bubble model seems more appropriate. The growth of a cloud depends on the entrainment across the interface identifying the cloud. The experiments of Redondo et al. (1995) model this growth in a non-homogeneous turbulent environment, studying the individual importance of the buoyancy induced or internal turbulence and the environmentally induced or external turbulence. Finally, in engineering, turbulent plumes are involved in building ventilation processes to supply fresh and cool air and are essential to evaluate quality of air in rooms (Nielsen, 1993).

A forced plume is generated by the steady release of mass, momentum and buoyancy from a source situated in a region of uniform or stably stratified ambient fluid (Morton, 1959). 
Initially, for a forced plume the driven mechanism is the momentum flux and later the buoyancy flux governs the dynamics (Morton, 1959). When momentum effects are more important than density differences and buoyancy effects, the forced plume is a jet. Therefore, pure jets are flows with no buoyancy as opposed to entirely buoyancy-dominated pure plumes, which have no initial momentum. Therefore, forced plumes have the characteristics of both jets and plumes and these are special cases of forced plumes.

The most popular model to describe the plume dynamics is the one-dimensional steady-state model by Morton et al. (1956), which has been extensively applied to investigate the dynamics of natural and laboratory jets and plumes under various source conditions. Morton et al. (1956) investigated the rise of turbulent buoyant plumes from point sources into a motionless but neutral or stably stratified environment. Their key hypothesis consists in a global representation of turbulence achieved by introducing an entrainment coefficient $\alpha_{\mathrm{E}}$, assumed to be constant. The governing equations of motion may be reduced to a set of three coupled linear differential equations (conservation of mass, momentum, and buoyancy). In all cases, the results strongly depend on the value of the entrainment coefficient that appears indeterminable by theoretical investigations. Comparison of laboratory experiments on jets and plumes with the formalism of Morton et al. (1956) showed good agreement concerning the scaling laws governing the dynamics of the flows, but this model is not sufficient to explain all the experimental (Turner, 1986; Sreenivas and Prasap, 2000; Tate, 2002) and geophysical data about plumes (Carazzo et al., 2008). Therefore, an improved description of the turbulent entrainment is necessary, as the Carazzo et al. (2008) model presents where the entrainment coefficient varies as a function of buoyancy and source distance. It is also necessary to understand the effects of source condition variations on the entrainment coefficient.

The purpose of this paper is to study the time evolution of the entrainment coefficient $\alpha_{\mathrm{E}}$ for a given downwards plume. We also investigate the variation of this time evolution under different source conditions. Additionally, we present the vertical and entrainment velocity fields to analyze their time variation under different source parameters. In Sect. 2, we describe the entrainment coefficient definition and a brief resume of values and entrainment models. In Sect. 3, we present our experimental configuration and procedures, qualitatively describing the experiments. In Sect. 4, we present our measurements of the plume vertical velocity and entrainment velocity fields and the entrainment coefficient. In Sect. 5, we present the discussion of results, studying the variation in time and under different source conditions of vertical velocity, entrainment velocity and entrainment coefficient. Finally, we briefly present our conclusions.

\section{Simple plume entrainment model}

We briefly resume the governing equations in a simple uniform ambient for a forced plume. We use an axisymmetric assumption and take cylindrical polar coordinates $(z, \theta, r)$ with the $z$ axis vertical and the source at the origin. We suppose that $\rho_{\mathrm{s}}, W$, and $U$ are the time-averaged source density, axial (known as vertical velocity) and radial velocities. We also consider the Atwood number $A$ that measures the density difference (therefore, the buoyancy) across the interface between the two fluids: the plume and the ambient fluid, $\rho_{\mathrm{a}}: A=\frac{\rho_{\mathrm{s}}-\rho_{\mathrm{a}}}{\rho_{\mathrm{s}}+\rho_{\mathrm{a}}}$.

We also define the mass flux $Q$, the buoyancy flux $B$ and the momentum flux $M$ as follows:

$Q=\rho_{\mathrm{s}} W b^{2}, \quad B=g\left(\rho_{\mathrm{a}}-\rho_{\mathrm{s}}\right) W b^{2}, \quad M=\rho_{\mathrm{s}} W^{2} b^{2}$,

where $g$ is the gravity acceleration and $b$ is the plume radius. We may express the buoyancy flux $B$ in terms of the Atwood number, noting that a basic simplification may be used: the source density $\rho_{\mathrm{s}}$ has a much greater (or smaller for negative buoyancy) density than the ambient fluid $\rho_{\mathrm{a}}$; then

$\rho_{\mathrm{S}} \gg \rho_{\mathrm{a}} \Rightarrow A=\frac{\triangle \rho}{\bar{\rho}}=\frac{\triangle \rho}{\rho_{\mathrm{s}}} \Rightarrow B=g \cdot A \cdot V$

where $V$ is the volume flux, $\Delta \rho=\rho_{\mathrm{s}}-\rho_{\mathrm{a}}$ and $\bar{\rho}=\frac{\rho_{\mathrm{s}}+\rho_{\mathrm{a}}}{2}$, which represents a reference density. However, in the case that their densities are similar:

$\rho_{\mathrm{s}} \approx \rho_{\mathrm{a}} \Rightarrow A=\frac{\triangle \rho}{2 \bar{\rho}} \Rightarrow B=2 \cdot g \cdot A \cdot V$.

The following equations are based on the same integrated equations of motion as the earlier work of Morton (1956) and Morton (1971). The same assumptions are made, which may be briefly restated as follows: plume is steady on timescale longer than eddy turnover time; the Boussinesq approximation holds throughout the flow and the profiles of mean vertical velocity and mean density deficiency remain similar at all plume cross-sections (at all heights) or self-similarity assumptions. Considering the mentioned definitions and the assumptions detailed above, using top-hat profiles to represent values of mean vertical velocity and mean buoyancy and applying conservation of mass, momentum and buoyancy, we obtain the following set of differential equations in the situation of a constant ambient density or neutral environment (then the Brunt-Väissäla frequency $N$ is zero as in our experimental configuration):

$\frac{\mathrm{d} Q}{\mathrm{~d} z}=2 \varrho^{1 / 2} \alpha_{\mathrm{E}} M^{1 / 2}, \frac{\mathrm{d} M}{\mathrm{~d} z}=\frac{B Q}{M}, \frac{\mathrm{d} B}{\mathrm{~d} z}=0$.

\subsection{The entrainment coefficient}

Stommel (1947) studied for the first time the entrainment concept. He studied the dilution of a cumulus cloud by entrainment of environmental air. 
An important further refinement of the entrainment formulation was pointed out by Houghton and Cramer (1951). They made a distinction between dynamical entrainment due to larger scale organized inflow (advective transport across the interface) and turbulent entrainment caused by turbulent mixing at the cloud edge (diffusive nature and described with an eddy diffusivity approach).

More precise quantitative descriptions of entrainment originated from laboratory water tank experiments of thermal plumes (Morton et al., 1956; Turner, 1962) that described an increasing mass flux with height. The one-dimensional model by Morton et al. (1956) establishes the linear growth of radius with distance from the source, which implies that the mean entrainment velocity is proportional to a characteristic upward or downward velocity. This statement is called the entrainment coefficient assumption and was first used by Morton et al. (1956) in the analysis of plumes. This key hypothesis consists in a global representation of turbulence achieved by introducing an entrainment coefficient $\alpha_{\mathrm{E}}$, assumed constant, that defines the horizontal rate of entrainment of surrounding fluid or entrainment velocity $U_{\mathrm{E}}$ in terms of the vertical velocity $W: U_{\mathrm{E}}=\alpha_{\mathrm{E}} W$ (Fig. 1). Therefore, it is possible to calculate the entrainment coefficient as $\alpha_{\mathrm{E}}=U_{\mathrm{E}} / W$. Closure of the equations is then possible through the use of the entrainment constant $\alpha_{\mathrm{E}}$.

Laboratory experiments have been performed to determine a range for $\alpha_{\mathrm{E}}$. Morton et al. (1956) did some experiments and they found a constant value of $\alpha_{\mathrm{E}}=0.13$, and proposed this value as a universal constant. In the absence of a theory for $\alpha_{\mathrm{E}}$, it has become common in geophysical problems to use an arbitrary value of 0.09 without a clear physical basis. However, decades of experiments on plumes and jets do not support the assumption of a universal constant value for $\alpha_{\mathrm{E}}$. In fact, most authors use different entrainment coefficients for different discharge and/or environmental conditions. For example, in Tate (2002), Wright (1984) uses an entrainment coefficient of 0.30 for discharges of a jet issuing from an axisymmetric source under conditions of stratified and stagnant ambient waters, and a value of 0.14 for discharges of a plume under the same conditions. The estimates of $\alpha_{\mathrm{E}}$. The entrainment coefficient $\alpha_{\mathrm{E}}$ also varies between 0.05 and 0.12 according to the direction of the buoyancy force, negatively or positively buoyant (Kaminski et al., 2005). In case of negatively buoyant jets the variation in $\alpha_{\mathrm{E}}=0.075 \pm 0.05$ is rather large as for plumes. In lazy plumes, $\alpha_{\mathrm{E}}$ is found to be around 0.12 (Hunt and Kaye, 2001). There appears to be considerable variability in the values of the entrainment coefficient as Tate (2002) presents in his work. For more details about values for the entrainment coefficient see tables 3.4-3.7 of its work. Tate (2002) summarizes values for the entrainment coefficient for different model types that can be found in the literature and classified depending on the experimental conditions (axisymmetric or line source, jet or plume, stratified or unstratified ambient fluid, and flowing or stagnant ambient fluid). For axisymmetric jets in an unstratified and stag-

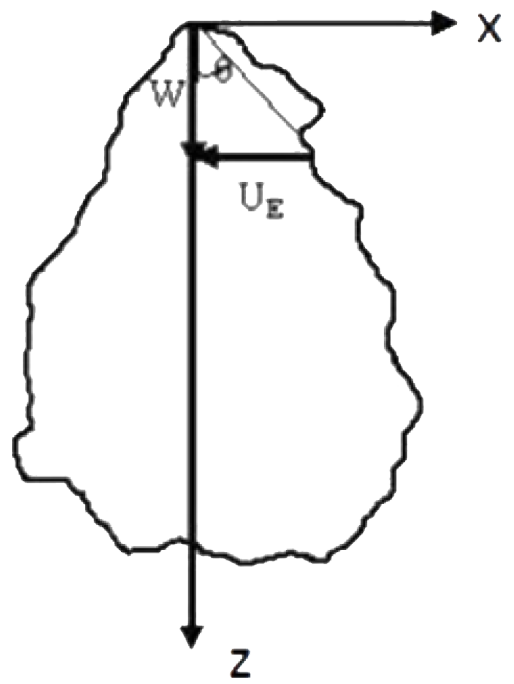

Fig. 1. Schematic view of a forced downwards plume without environmental turbulence. The mean centerline speed is the vertical velocity $W$ and radius $b$. The plume entrains ambient fluid characterized by a mean entrainment velocity $U_{\mathrm{E}}$. The figure shows the angle of the plume $\theta$.

nant ambient fluid, the mean entrainment is $0.080 \pm 0.029$, and for axisymmetric plumes under the same conditions the mean entrainment is $0.110 \pm 0.034$.

Therefore, the main limitation of Morton's model is its assumption of a constant value for $\alpha_{\mathrm{E}}$. As a consequence this model cannot explain that the values of $\alpha_{\mathrm{E}}$ for a jet and a plume differ significantly, varying between 0.05 and 0.16 , respectively (Kaminski et al., 2005), so a forced plume cannot be characterized by a single value, a constant entrainment coefficient can only give approximate predictions (at first it will exhibit jet-like characteristics and ultimately it must exhibit plume-like characteristics).

Frick (1984) recognized that a more complex entrainment function is necessary in order to reproduce the results in laboratory and field experiments. Instead of using $W=U_{\mathrm{E}} / \alpha_{\mathrm{E}}$, he establishes a difference between the shear entrainment, $\left(\alpha_{\mathrm{E}}\right)_{\text {shear }}$, and the vortex entrainment, $\left(\alpha_{\mathrm{E}}\right)_{\text {vortex }}$. Until recently, there was no theoretical explanation for the variation of $\alpha_{\mathrm{E}}$. The model of Kaminski et al. (2005) explains these variations and the role of both positive and negative buoyancy in the entrainment process is highlighted. The larger values of $\alpha_{\mathrm{E}}$ in pure and lazy plumes are due to positive buoyancy which enhances the entrainment of background fluid by promoting the formation of large-scale turbulence structure. Conversely, a negative buoyancy force inhibits entrainment and reduces $\alpha_{\mathrm{E}}$ (Kaminski et al., 2005). On the other hand, the variations within pure jets and pure plumes can be explained by the downstream evolution of each flow to a state of self-similarity (Carazzo et al., 2006).

Experimental measurements in forced plumes have shown that turbulent entrainment is limited in the near source part 
of the flow where inertial forces dominate compared with the buoyancy-dominated region far from the source (Morton, 1959). To account for this evolution, some authors proposed empirical or semi-empirical parametrizations allowing $\alpha_{\mathrm{E}}$ to vary according to the ratio of the buoyancy and inertia forces:

$\alpha_{\mathrm{E}}=\alpha_{\mathrm{j}}-\left(\alpha_{\mathrm{j}}-\alpha_{\mathrm{p}}\right)\left(\frac{F r_{\mathrm{p}}}{F r}\right)^{2}$,

where $F r$ is the Froude number and $F r_{\mathrm{p}}$ is the constant Froude number for a pure plume in a uniform environment, and $\alpha_{\mathrm{j}}$ and $\alpha_{\mathrm{p}}$ are arbitrary constant values of $\alpha_{\mathrm{E}}$ for pure jets and pure plumes, respectively (Lee and Chu, 2003). The values of the entrainment coefficient that best fit the data fall between the bounds formed by the values of $\alpha_{\mathrm{E}}$ for pure jets and pure plumes in uniform environments (Carazzo et al., 2006) except at large distances from the source where $\alpha_{\mathrm{E}}$ values are even smaller than those predicted for a pure jet.

The variable entrainment models may easily be adapted to a good agreement with small-scale laboratory experiments, with the Morton et al. (1956) model obtaining reasonable predictions at both small and at large distances from the source. On the other hand, they are also useful with geophysical or environmental turbulent plumes such as volcanic plumes on Earth or other planets, atmospheric sources of pollution, sewer discharge plants near the coast or submarine plumes rising from hydrothermal vents.

\section{Experimental setup}

The aim of the experimental procedure is to generate a turbulent axysimmetric plume, descending from a finite source in an unstratified and stagnant environment fluid and controlling its position and its physical characteristics as buoyancy and momentum fluxes (see Table 1). The experiments consisted of releasing a denser fluid downwards from a small nozzle, with a diameter $D$ of $0.6 \mathrm{~cm}$ and an area $S_{0}$ of $0.2827 \mathrm{~cm}^{2}$, into a stationary body of water that was contained in a glass tank of dimensions $32 \mathrm{~cm}$ high by $25 \mathrm{~cm}$ by $25 \mathrm{~cm}$. This water layer is designated as the lighter layer with a height $h_{\mathrm{L}}=17 \mathrm{~cm}$ and a density $\rho_{\mathrm{L}}=1.0 \mathrm{~g} \mathrm{~cm}^{-3}$. On top of this lighter layer, a system made of two metacrylic boxes is placed (Fig. 2). The bottoms of the boxes are pierced with one regulated orifice that is at a height $H_{\mathrm{o}}$ from the lighter layer and represents the position of the injection orifice. These boxes contain the denser layer that reaches a height $h_{\mathrm{D}}=1.134 \mathrm{~cm}$ and a density $\rho_{\mathrm{D}}$ (López, 2004; López et al., 2008). The starting point of the plume is located at a height $H_{\mathrm{o}}$, which takes the values $0,2,3,6$, and $8 \mathrm{~cm}$. For each $H_{\mathrm{o}}$, we use four different potassium permanganate solutions as the releasing fluids with an intense purple color and a volume of $500 \mathrm{~cm}^{3}$. This provides the following range of Atwood numbers: 0.001, 0.0025, 0.005, and 0.01. Denser fluid with momentum and buoyancy fluxes is discharged from the orifice continuously at a flow rate of $V_{\mathrm{o}}=8.40 \mathrm{~cm}^{3} \mathrm{~s}^{-1}$. A
Table 1. The 16 different experimental conditions of the fluid system. The density of the denser layer is $\rho_{\mathrm{D}} . A$ is the Atwood number. The location of the ejecting orifice is $H_{\mathrm{O}}$. The buoyancy and momentum fluxes at the impinging surface are $B_{1}$ and $M_{1}$.

\begin{tabular}{ccccc}
\hline $\begin{array}{c}\rho_{\mathrm{D}} \\
\left(\mathrm{g} \mathrm{cm}^{-3}\right)\end{array}$ & $A$ & $\begin{array}{c}H_{\mathrm{O}} \\
(\mathrm{cm})\end{array}$ & $\begin{array}{c}M_{1} \\
\left(\mathrm{~cm}^{4} \mathrm{~s}^{-2}\right)\end{array}$ & $\begin{array}{c}B_{1} \\
\left(\mathrm{~cm}^{4} \mathrm{~s}^{-3}\right)\end{array}$ \\
\hline \multirow{2}{*}{1.002} & 0.001 & 3 & 1911.869 & -22783.376 \\
& & 6 & 3574.145 & -31151.221 \\
& & 8 & 4682.329 & -35654.960 \\
\hline \multirow{2}{*}{1.005} & 0.0025 & 3 & 1357.777 & -19200.089 \\
& & 6 & 3574.145 & -31151.221 \\
1.0101 & 0.005 & 3 & 4682.329 & -35654.960 \\
& & 6 & 3574.145 & -31151.221 \\
& & 8 & 4682.329 & -35654.960 \\
\hline \multirow{3}{*}{1.0202} & 0.010 & 3 & 1911.869 & -22783.376 \\
& & 6 & 3574.145 & -31151.221 \\
& & 8 & 4682.329 & -35654.960 \\
\hline
\end{tabular}

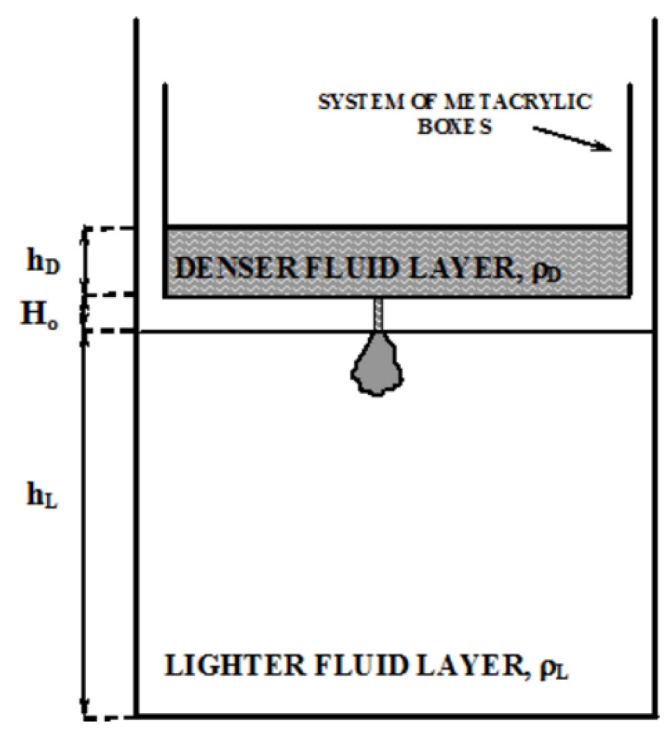

Fig. 2. Schematic diagram of the experimental configuration that shows the initial state of the fluid system with a starting forced plume. $h_{\mathrm{L}}$ and $\rho_{\mathrm{L}}$ are the height and the density of the lighter layer of fluid; $h_{\mathrm{D}}$ and $\rho_{\mathrm{D}}$ are the height and the density of the denser layer and the height $H_{\mathrm{O}}$ is the source position and varies from 0 to $8 \mathrm{~cm}$.

sketch of the experimental setup is given in Fig. 2 (López, 2004; López et al., 2008).

It is not necessary to add a dye as a passive tracer and, therefore, the flow can be directly visualized (back illuminated). The video recordings at high-velocity mode (100 fps) 
of the experiments are sequenced into frames using framesequencer software ( 640 by 480 pixels capturing an area of 25 by $18 \mathrm{~cm}$ ). The registered images average the concentration over the volume of the plume.

Table 1 lists the characteristics of the 16 different experimental conditions of the fluid system and the generated plume. The rate of supply of buoyancy is the buoyancy flux of the plume at source $B_{0}$, whose value is $-8232 \mathrm{~cm}^{4} \mathrm{~s}^{-3}$. The buoyancy flux is negative because the discharge fluid is heavier than the ambient fluid. The rate of supply of momentum by the source is the momentum flux at source $M_{\mathrm{o}}$ whose value is $249.593 \mathrm{~cm}^{4} \mathrm{~s}^{-2}$. The denser fluid is injected through the orifice with these fluxes and impinges on the lighter layer. $B_{1}$ and $M_{1}$ are the initial effective buoyancy and momentum fluxes at the free surface of the lighter layer as a consequence of the conversion from potential energy to initial kinetic energy of the plume.

\subsection{Plume growth in steady environments}

Plume occurs where fluid of different density from the ambient fluid is injected, as the density of the effluent is heavier than its surrounding ambient fluid and, therefore, the buoyancy force is downward and the initial flow is directed vertically downwards in the same direction as the buoyancy forces. Figure 3 shows several sequences of digitized video images from two experiments under different source conditions where $t^{*}$ is the non-dimensional time defined as $t^{*}=\frac{t}{t_{\mathrm{c}}}$. The timescale $t_{\mathrm{c}}$ is the time that a plume spends in crossing the distance $H_{\mathrm{o}}+h_{\mathrm{L}}$ (see Fig. 2): $t_{\mathrm{c}}=\sqrt{\frac{H_{\mathrm{O}}+h_{\mathrm{L}}}{A g}}$.

Upon entering the ambient fluid flow, the denser fluid becomes unstable and forms the axisymmetric plume at the center of the tank. As the plume is gravitationally unstable, it engulfs lighter fluid as it evolves, but the forced plume has different behaviors: jet-like characteristics depending on its initial mass and momentum fluxes, and plume-like characteristics depending on its initial buoyancy flux. If we use the Fischer characteristic length $L_{\mathrm{m}}$ (Fischer et al., 1979) and its related dimensionless parameter $z / L_{\mathrm{m}}$, it is possible to analyze the degree of jet-like and plume-like behaviors of the forced plume. Our Fischer's length ranges from 10.94 for the smallest Atwood number $(A=0.001)$ to 3.46 for the greatest Atwood number $(A=0.01)$.

A forced plume can be considered as a pure jet at $\frac{z}{L_{\mathrm{m}}}<0.5$ and as a pure plume at $\frac{z}{L_{\mathrm{m}}}>5$; when $0.5<\frac{z}{L_{\mathrm{m}}}<5$ we have the transition of the forced plume (Fischer et al., 1979). For our plume experiments, $z / L_{\mathrm{m}}$ ranges from 0.165 (for the smallest $z$, Atwood number $A$ and initial Richardson number $R i_{\mathrm{o}}$ ) to 1.879 (for the greatest $z, A$, and $R i_{\mathrm{o}}$ ). Then, the dynamical behavior of the fluid flow modifies from a pure jet-like to a forced plume in a transition state. Moreover, for a given experiment or fixed buoyancy and source position, the non-dimensional quantity $z / L_{\mathrm{m}}$ increases with time.

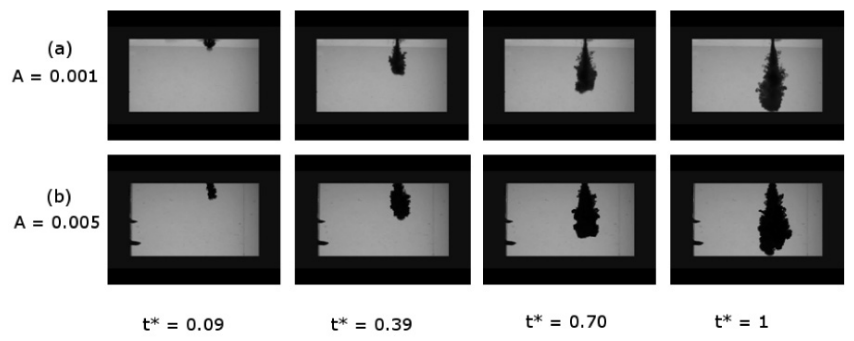

Fig. 3. Time evolution of one turbulent plume through its frame sequences corresponding to two experiments with different Atwood numbers $A$ made with the same height for the starting point $H_{\mathrm{O}}=3 \mathrm{~cm}$. (a) $A=0.001$ and (b) $A=0.005$ for non-dimensional time $t^{*}=0.09,0.39,0.70$, and 1 .

Buoyancy is said to be dominant after some distance, which verifies $z>5 L_{\mathrm{m}}$, which is different for each experiment. Usually, after ten to twenty source diameters the buoyancy dominates the flow. At this stage the entrainment of the ambient fluid directed through the border of the turbulent plume is more effective. The sides of the plume are zones with strong shears, which generate Kelvin-Helmholtz instabilities, and secondary Rayleigh-Taylor instabilities may also appear at the front of the convective plume.

Finally, the plume advance spreads sideways as it evolves until it reaches the physical contours of the tank which limit the development of the plume generating a gravity current and an overturn of the fluid system.

\section{Quantitative results: velocity fields and entrainment coefficient variations}

We use the PIV (particle image velocimetry) method to understand the behavior of turbulent plume based on our velocity field measurements. We calculate velocity PDFs with the DigiFlow program (Matulka et al., 2008) using sequences from experiments that were videotaped. We analyze the evolution of the velocity fields visualizing different graphics, which can give us an idea about vertical velocity, the entrainment velocity and the entrainment coefficient. The study of the velocity fields gives us information about the time progress of the turbulent plume, in which direction is the propagation of the plume or with what velocity is flowing. We can find its vertical velocity by looking at the length of the plume or entrainment velocity by looking at the width of the plume.

We calculate the vertical velocity as $W=z / t\left[\mathrm{cms}^{-1}\right]$, where $z$ is the length of the turbulent plume and $t$ is the time of the propagation of the plume. The magnitude $z_{\mathrm{i}}$ measures the length of the plume in each time $t_{\mathrm{i}}$. On the velocity fields we can see velocity vectors and their directions; the size of the vectors depends on the initial velocity of the plume. Figure 4 shows the elements for the vertical velocity calculation for a selected plume experiment with $A=0.001$ and $H_{\mathrm{o}}=2 \mathrm{~cm}$. 


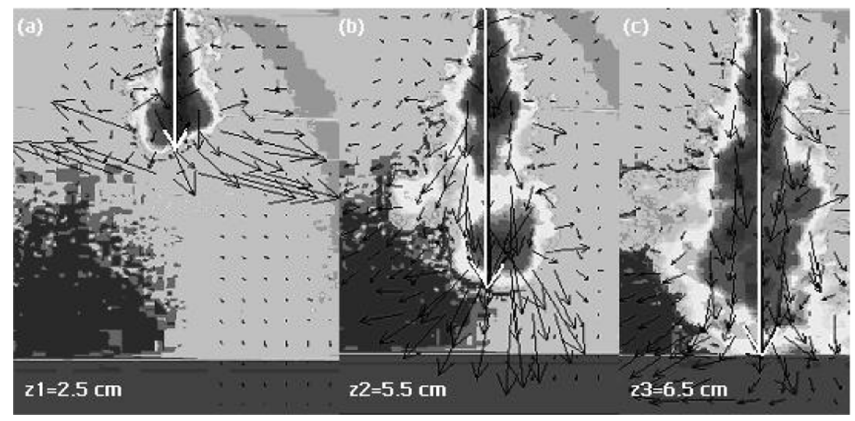

Fig. 4. Time sequence of a turbulent plume experiment with Atwood number $A=0.001$ and $H_{0}=2 \mathrm{~cm}$. The values of $z_{1}, z_{2}$, and $z_{3}$ are shown. (a) Plume at $t_{1}^{*}=0.2$, (b) plume at $t_{2}^{*}=0.5$, and (c) plume at $t_{3}^{*}=1.0$.

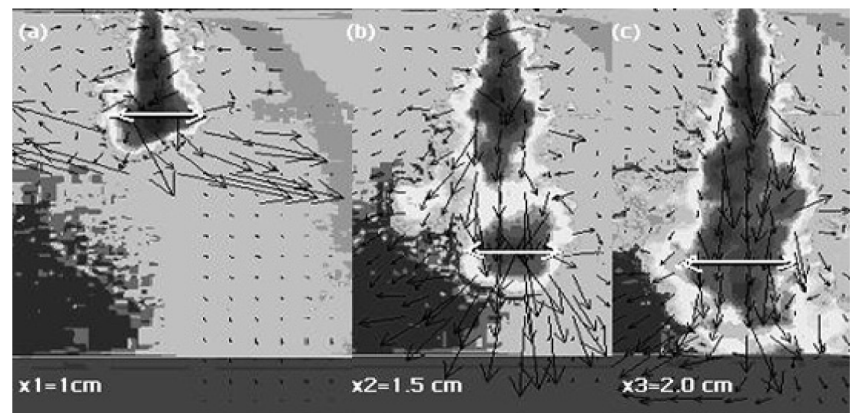

Fig. 5. Time sequence of a turbulent plume experiment with Atwood number $A=0.001$ and $H_{0}=2 \mathrm{~cm}$. The values of $x_{1}, x_{2}$, and $x_{3}$ are shown. (a) Plume at $t_{1}^{*}=0.2$, (b) plume at $t_{2}^{*}=0.5$, and (c) plume at $t_{3}^{*}=1.0$.

We calculate entrainment velocity as $U_{\mathrm{E}}=x / t\left[\mathrm{~cm} \mathrm{~s}^{-1}\right]$ where the $x$ is the width of the plume at the maximum point of it, and $t$ time is the same as for the vertical velocity. Figure 5 shows the elements for the entrainment velocity calculation for the same selected plume as in Fig. 4. Therefore, it is possible to calculate the entrainment coefficient as defined in Sect. 2.1.

As a consequence of this procedure, it is possible to analyze the time evolution of the vertical velocity $W$, the entrainment velocity $U_{\mathrm{E}}$ and the entrainment coefficient $\alpha_{\mathrm{E}}$. Figure 6 shows the time evolution of the vertical velocity $W$ under different source conditions (varying Atwood numbers with a fixed $H_{\mathrm{o}}$ and source positions $H_{\mathrm{o}}$ with a fixed Atwood number).

The time variation of vertical velocity has a greater dispersion at early times (Fig. 6) and $W$ ranges from 12 to $27 \mathrm{~cm} \mathrm{~s}^{-1}$. Close to the end of the experiment, the vertical velocity has no dispersion and tends to a constant value close to $6.5 \mathrm{~cm} \mathrm{~s}^{-1}$ independently of Atwood number $A$ and $H_{\mathrm{o}}$. This limit vertical velocity does not depend on source conditions. Therefore, differences are only evident at early times and there are no differences at the end of the experiments for all source conditions.

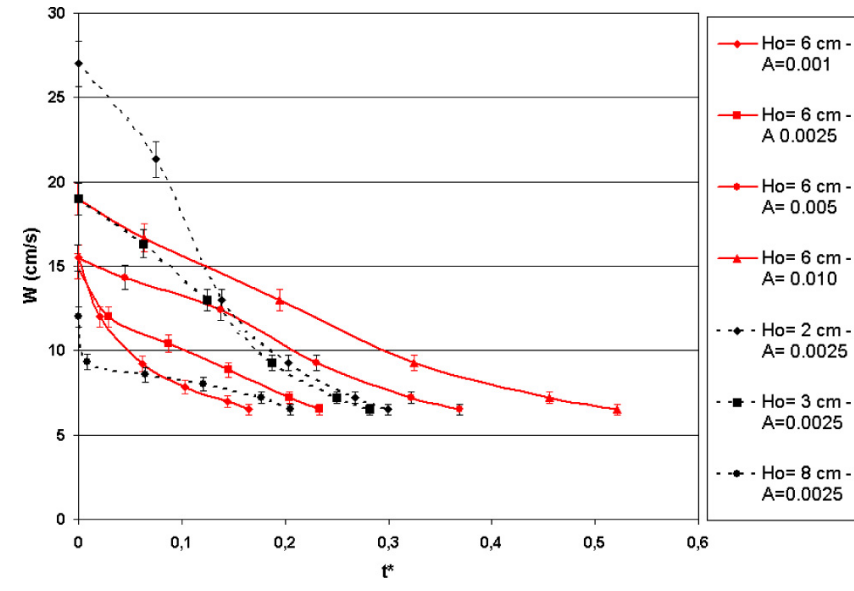

Fig. 6. Time evolution of the vertical velocity $W$ under different source conditions.

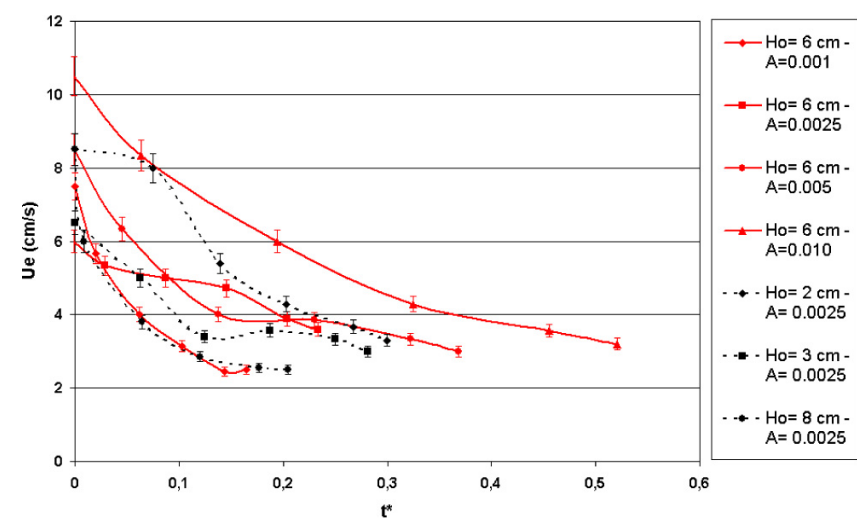

Fig. 7. Time evolution of the entrainment velocity $U_{\mathrm{E}}$ under different source conditions.

The entrainment velocity also has dispersion at initial times (Fig. 7), but the time behavior is slightly different if we compare it with the evolution of the vertical velocity, especially at the end of the experiment. The dispersion at early times ranges from 6 to $10.5 \mathrm{~cm} \mathrm{~s}^{-1}$ and it ranges from 2.5 to $3.3 \mathrm{~cm} \mathrm{~s}^{-1}$ at the end of the experiment. We observe that the data dispersion decreases in time. We observe that the entrainment velocity decreases in time in a similar way for all the source conditions (Fig. 7). The most evident difference is that there is no limit value for $U_{\mathrm{E}}$ close to the end of the experiment: different values of the source conditions ( $A$ and $H_{\mathrm{o}}$ ) have different $U_{\mathrm{E}}$.

The entrainment coefficient also has a marked dispersion at initial times (Fig. 8) and is less important close to the end of the experiment. At early times, the entrainment coefficient has an important dispersion and ranges between 0.40 and 0.55 for variable Atwood number experiments and between 0.31 and 0.71 for variable $H_{\mathrm{o}}$ experiments. Close to the end of the experiment, the entrainment coefficient ranges between $(0.39,0.55)$ for the first kind of experiments and 


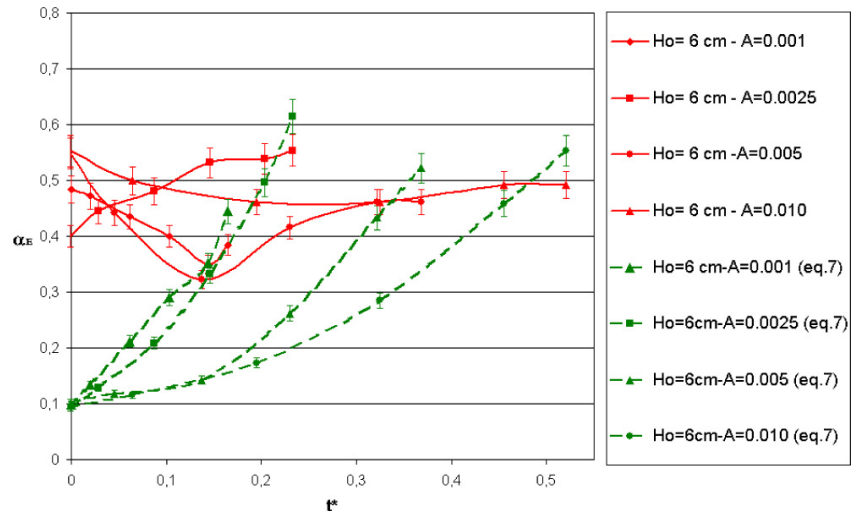

(a)

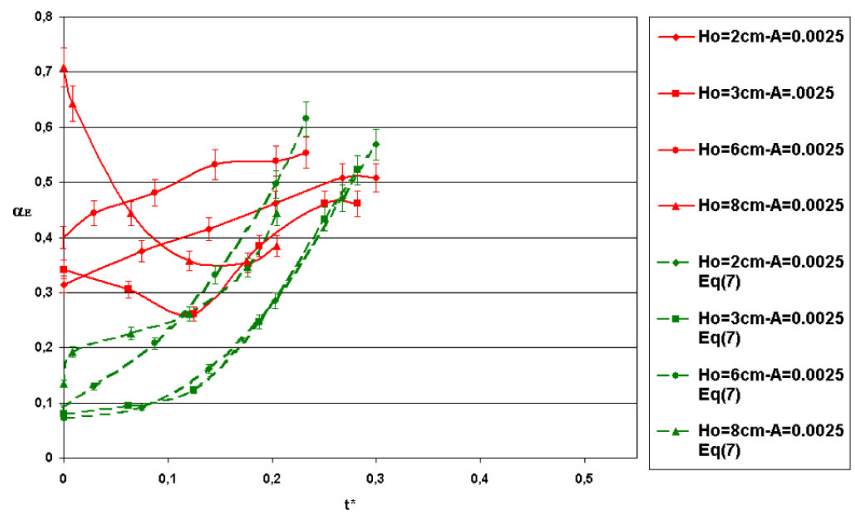

(b)

Fig. 8. Time evolution of the entrainment coefficient $\alpha_{\mathrm{E}}$ under different experimental conditions. (a) With a fixed $H_{\mathrm{O}}=6 \mathrm{~cm}$; dashed lines represent the evaluation of Eq. (7) for the same source conditions. (b) With a fixed Atwood number $A=0.0025$; dashed lines represent the evaluation of Eq. (7) for the same source conditions.

$(0.39,0.55)$ for the second ones. Therefore, the entrainment coefficient $\alpha_{\mathrm{E}}$ does not tend to a constant value and shows a time evolution within the same plume and for all the source conditions.

A verification that the time evolution of the entrainment coefficient exits within the same plume is to measure the angle of the plume because of the following relation $\alpha_{\mathrm{E}} \approx$ $\tan (\theta)$ (Fig. 1). Figure 9 shows the time evolution of the plume angle for an experiment with $H_{\mathrm{o}}=2.5 \mathrm{~cm}$ and $A=$ 0.03 . From these data of a measured angle, $\theta$, the following empirical linear fit is deduced: $\theta=8.34+10.27 t^{*}$. The value of the coefficient of determination $R^{2}$ is 0.75 , which indicates that the presented linear model explains $75 \%$ of the variability in our angle data set. From the same data of the plume angle it is possible to deduce a better non-linear fit that is a $\log$ fit $\theta=16.86+3.40 \log \left(t^{*}\right)$. Figure 9 shows this $\log$ fit (dashed line). This non-linear log model explains $91 \%$ of the variability in our angle data set. Therefore, the angle of the plume angle that is indirectly related to the entrainment coefficient is not constant in time.

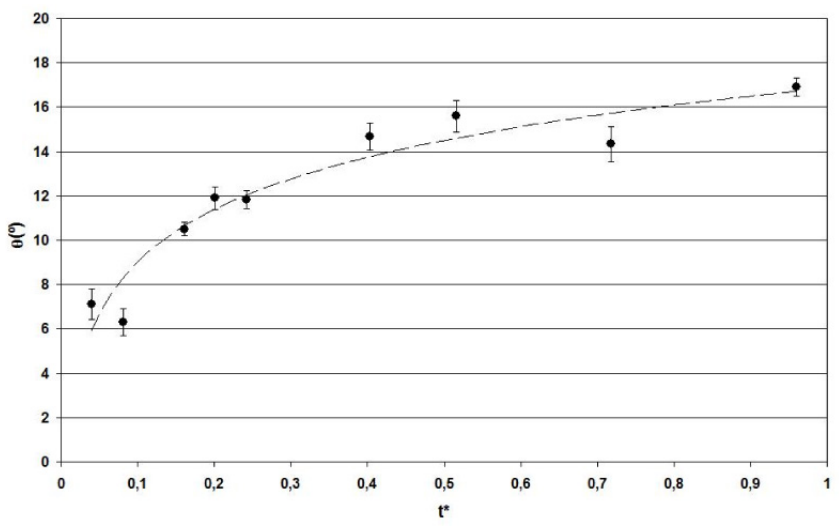

Fig. 9. Time evolution of the angle $\theta\left(^{\circ}\right)$ of the plume for an experiment with a fixed $H_{\mathrm{O}}=2.5 \mathrm{~cm}$ and Atwood number $A=0.03$. The $\log$ fit (dashed line) is also shown.

\section{Brief discussion and future work}

The original model of Morton et al. (1956) is not sufficient to explain all the experimental and geophysical data about plumes, as was clearly stated in Sect. 2.1. The present laboratory experimental work was motivated by the idea of verifying if the entrainment coefficient varies in time within the same plume and changes with source conditions. Our results about the behavior of the entrainment coefficient verify all these assumptions.

We have considered the topological and geometrical effects affecting the initial evolution of jets/plumes that also appear in nature. The role of ambient turbulence and of environmental buoyancy as discussed by Redondo et al. (1995) and Redondo and Yagüe (1994) will be considered in future work. Here we present an experimental study where we measure the entrainment coefficient, analyzing its time behavior within the same plume and under different source conditions (Fig. 8). We also study the behavior of vertical and axisymmetric velocity at early times under different source conditions (Figs. 6 and 7).

In general, vertical velocities are greater for variable $H_{\mathrm{o}}$ experiments (Fig. 6), i.e., the source position $H_{\mathrm{o}}$ has an effect on vertical velocity. Close to the end of the experiment, there is a limit to the vertical velocity for the plume $\left(6.5 \mathrm{~cm} \mathrm{~s}^{-1}\right)$ that is not affected by the buoyancy $A$ and the location of the ejection orifice $H_{0}$, i.e., it is independent of the source conditions. The model of Morton et al. (1956) gives the mean vertical speed of a plume $W \propto \frac{1}{b^{1 / 3}}$ where $b$ is the radius of the plume under the assumption of a constant entrainment coefficient. Therefore, if the radius grows, the plume evolves in time, goes downwards and its vertical velocity decreases. This speed decrease is not affected by the source conditions after local equilibrium at the top-heavy front is attained (Fig. 6). 
The behavior of the entrainment velocity is represented by Fig. 7. The entrainment velocity decreases in time in a similar way for all source conditions. Given the definition of the entrainment coefficient and the relation for the mean vertical speed of the model of Morton et al. (1956), it is possible to suppose that the entrainment velocity decreases in a similar way $\left(U_{\mathrm{E}} \propto \frac{1}{b^{1 / 3}}\right)$ if the entrainment coefficient is supposed constant. For these experiments, there is no clear limiting value for $U_{\mathrm{E}}$ close to the end of the experiment. For variable Atwood number experiments, the entrainment velocity $U_{\mathrm{E}}$ increases with the Atwood number. As the position of the orifice $H_{\mathrm{o}}$ is fixed, only the buoyancy changes by means of the Atwood number $A$; therefore, the increase in buoyancy increases the entrainment velocity. For variable $H_{\mathrm{O}}$ experiments, the entrainment velocity $U_{\mathrm{E}}$ also decreases in time (Fig. 7). As buoyancy is fixed, only the source location changes, but the entrainment velocity does not clearly increase with the variation in the source location.

Finally, we conclude that the entrainment velocity increases with the Atwood number, $A$, and it is not so sensitive to the source location, $H_{0}$.

In this turbulent plume study we have observed that the entrainment coefficient has important variations in time within the same plume and also with the initial buoyancy and source position conditions, as Fig. 8 shows. At early times, the entrainment coefficient has an important dispersion and close to the end of the experiment, the dispersion of the entrainment coefficient is less important, as in all cases the behavior is plume-like. Although the dispersion has decreased in time, it is clear that the entrainment coefficient $\alpha_{\mathrm{E}}$ does not tend to a constant value and has a time evolution within the same plume and for all the source conditions. This is our main result, which differs from other authors who compare different plumes or jets, or plumes and jets.

This result has an important theoretical implication because in generating the asymptotic equations for a buoyant fluid discharged into an ambient fluid (may be flowing or stagnant, stratified or unstratified), the entrainment coefficient $\alpha_{\mathrm{E}}$ is assumed to be independent of time (Tate, 2002). Therefore, if a time variation for $\alpha_{\mathrm{E}}$ is confirmed, the mathematical treatment to deduce the asymptotic equations should change to introduce a time dependence in the entrainment coefficient function. More experimental work is necessary to look for this time dependence and to achieve this aim, and in particular to understand the important differences between axisymmetric plumes within a 3-D space and line plumes that exhibit a 2-D geometry.

Figure $8 \mathrm{~b}$ shows the time behavior of the entrainment coefficient for variable $H_{\mathrm{o}}$ experiments. As Atwood number $A$ is constant, the effect of buoyancy is constant and only the effect of the source location is considered. The behavior of the entrainment coefficient is non-regular in time and varies with the source position, $H_{0}$. As the location of the source is higher, the coefficient $\alpha_{\mathrm{E}}$ is greater at early time and also at the end of the experiment.
Moreover, the measured entrainment coefficient has considerable variability, more evident at initial steps of the experiments. We also observe that the entrainment coefficient values are greater than the typical value of Morton et al. (1956) and the values for plumes (between 0.085 and 0.16 ) and jets (between 0.051 and 0.07). Considering all the results, we have evaluated Eq. (5) using our data. To evaluate the equation we write the Froude number $\mathrm{Fr}$ in terms of the Atwood number $A$. The Froude number $F r$ is a dimensionless quantity that measures the relative importance of the inertial and gravitational effects on a fluid flow. The local densimetric Froude number of a plume based on the centerline values of the vertical velocity and reduced gravity is:

$$
F r=\frac{W}{\sqrt{\frac{\Delta \rho}{\bar{\rho}} g b}}=\frac{W}{\sqrt{2 A g b}},
$$

where $\Delta \rho$ is the density difference between plume and ambient fluids $\left(\rho_{\mathrm{D}}-\rho_{\mathrm{L}}\right)$ and $\bar{\rho}$ is the average fluid density. Therefore, Eq. (5) would be:

$\alpha_{\mathrm{E}}=\alpha_{\mathrm{j}}-\left(\alpha_{\mathrm{j}}-\alpha_{\mathrm{p}}\right)\left(\frac{2 F r_{\mathrm{p}}^{2} A g b}{W}\right)^{2}$,

where $F r_{\mathrm{p}}=4.5, \alpha_{\mathrm{j}}=0.057$ (for a round jet) and $\alpha_{\mathrm{p}}=0.09$ (for a round plume) (Lee and Chu, 2003). Equation (7) shows that the entrainment coefficient $\alpha_{\mathrm{E}}$ depends on the Atwood number $A$, the plume vertical velocity $W$ and the plume radius $b$.

Figure 8 shows the results of the evaluation of Eq. (7) for variable Atwood number $A$ (Fig. 8a, dashed lines) and variable source position $H_{\mathrm{o}}$ (Fig. 8b, dashed lines) experiments. For both kind of experiments, a similar behavior appears: the evaluated entrainment coefficient always increases in time. Therefore, Eq. (7) also predicts a time change for $\alpha_{\mathrm{E}}$. Another conclusion is that our experimental entrainment coefficient is clearly greater (and has more dispersion) than that evaluated by Eq. (7) at early times. Our greater values of $\alpha_{\mathrm{E}}$ could be related to the plume characteristics. The plume is not laminar and it presents turbulence from the starting point (with momentum and buoyancy fluxes). Another reason could be that the values of the parameters $\alpha_{\mathrm{j}}$ and $\alpha_{\mathrm{p}}$ in Eq. (7) are not the most adequate for our configuration. Our measurements are still greater close to the end of the experiments, but the difference in values is less important. Really, at the end of the experiment, all the values (the experimental ones and those evaluated from Eq. 7) range between 0.38 and 0.62 .

We analyze in more detail the behavior of entrainment. For environmental and complex situations the initial conditions may have either 2- or 3-D basic starting topologies. In both cases there is a jet to plume transition scale and the evolution of the starting jet/plume is always slower that the traditional uniform Rayleigh-Taylor instability of $z \propto t^{2}$ 
(López et al., 2008). As dimensions of volume, momentum and buoyancy fluxes for 2-D are $[Q]=L^{2} T^{-1},[M]=$ $L^{3} T^{-2},[B]=L^{3} T^{-3}$ and for 3-D they are $[Q]=L^{3} T^{-1}$, $[M]=L^{4} T^{-2},[B]=L^{4} T^{-3}$, then the respective jet-plume transition length scales are (Redondo and Yagüe, 1994):

$\mathrm{七}_{J P 2 D}=C_{2 D} M B^{2 / 3}, \mathrm{七}_{J P 3 D}=C_{3 D} M^{3 / 4} B^{-1 / 2}$.

So their mixing front time evolutions are $z \propto B^{1 / 3} t$ for 2$\mathrm{D}$ and $z \propto B^{1 / 4} t^{3 / 4}$ for 3-D. This is verified in Landel et al. (2012) as well as in Carazzo et al. (2006) and also indicates that Rayleigh-Taylor or top-heavy initial mixing can be very efficient.

In the case of turbulent or stratified environments, local entrainment values are strongly dependent on height and it is still necessary to evaluate higher order effects such as local variability, non-homogeneity and intermittency. It is interesting to use dimensional analysis to derive a general nondimensional parameter space for use in the environment because observations seldom correspond to equilibrium.

For 2-D or line sources we use $\frac{w}{U}, \frac{U^{3}}{N M}$ where $N$ is the buoyancy frequency, and for 3-D axisymmetric sources we have $\frac{w}{U}, \frac{U^{4} M}{B^{2}}$ or $\frac{U^{4}}{N B}$ as a density Froude number that expresses the relative importance of kinetic to potential energies. In both cases (2- and 3-D) $N M / B$ is a measure of the relative importance of momentum and buoyant fluxes in the presence of stratification. Finally, $U^{4} /\left(N^{2} M\right)$ represents the relative importance of the motion at buoyant fluid compared with an initial jet.

The non-dimensional parameter space is useful for generating a 4-D surface that represents the variation of the entrainment coefficient, because there is no constant value for $\alpha_{\mathrm{E}}$ (for a single experiment under the same conditions, the entrainment coefficient changes due to intermittency, changes from jet to plume or from a 2- to a 3-D situation). Further work should analyze intermittency and extend the entrainment assumptions to include higher order moments of the velocity and density moments and structure functions (Vindel at al., 2008) following an experimental procedure described in Mahjoub et al. (1998).

\section{Conclusions}

In this experimental study of a turbulent plume, our main result is that the entrainment coefficient is not a constant and clearly varies in time within the same plume independently of the buoyancy and the source position. Other authors compare different plumes or different jets, or plume and jet experiments, but our result is for a given plume experiment. We also observe that the time evolution of the entrainment coefficient appears with different initial experimental forcing conditions.

Moreover, the measured entrainment coefficient has considerable variability, more evident at initial steps of the experiments. We find as in Carazzo et al. (2006) that, at early times, the entrainment coefficient has an important dispersion and ranges between 0.26 and 0.9 for variable Atwood number experiments and between 0.16 and 0.55 for variable source position experiments. It is interesting to use these alternative descriptors instead of the usual momentum fluxes of volume, momentum and buoyancy because of an easier comparison with the highest initial mixing ability of the Rayleigh-Taylor instability (López et al., 2008). It is also observed that close to the end of the experiment, the dispersion of the entrainment coefficient is less important; it ranges between $(0.17,0.46)$ for variable Atwood number experiments and $(0.37,0.49)$ for variable source location experiments. The values of the coefficient are greater than the typical value of Morton et al. (1956) (0.13) and usually measured or calculated entrainment coefficients (but it is not an exception because List (1982) presents values of 0.57 and $(0.47,0.88)$, or Frick (1984) a value of 0.56).

It is equivalent to consider an initial condition parameter of $\left(Q_{\mathrm{o}}, M_{\mathrm{o}}, B_{\mathrm{o}}\right)$ or $\left(H_{\mathrm{o}}, V_{\mathrm{o}}, A_{\mathrm{o}}\right)$ to determine the initial effects including the angle of spread of the jet/plume, but the variability of ambient conditions seems also to need a complex and extended parameter space as discussed above. In terms of a generalized turbulent Schmidt or Prandtl number with values between 0.5 and 2, the jets (high $H_{\mathrm{o}}$ and $A$ ) reach their equilibrium conditions faster than the plumes, in agreement with Carazzo et al. (2006) for 3-D flows. In clear 2-D situations the sideways meandering of the jets/plumes increases the effective entrainment and the angles as indicated by Landel et al. (2012). Therefore it is necessary to provide more complex models of entrainment that depend on the jet to plume lengths for 2- and 3-D as well as other factors.

Acknowledgements. The authors would like to thank the ICMAT Severo Ochoa project SEV-2011-0087 for its financial support. The authors would also like to thank the help of M. García Velarde and the facilities offered by the Pluri-Disciplinary Institute of the Complutense University of Madrid. We also acknowledge the help of the European Community under project Multi-scale complex fluid flows and interfacial phenomena (PITN-GA-2008-214919). Thanks are also due to ERCOFTAC (PELNoT, SIG 14). We acknowledge the referees for assisting in evaluating this paper.

Edited by: A. M. Mancho

Reviewed by: P. Fraunie, M. Jellinek, and two anonymous referees

\section{References}

Carazzo, G., Kaminski, E., and Tait, S.: The route to self-similarity in turbulent jets and plumes, J. Fluid. Mech., 547, 137-148, 2006.

Carazzo, G., Kaminski, E., and Tait, S.: On the rise of turbulent plumes: Quantitative effects of variable entrainment for submarine hydrothermal vents, terrestrial and extra terrestrial explosive volcanism, J. Geophys. Res., 113, 1-19, 2008. 
Fischer, H. B., List, E. J., Koh, R. C., Imberger, J., and Brooks, N. H.: Mixing in inland and coastal waters, Academic Press Inc. (London) Ltd., 1979.

Frick, W. E.: Non-empirical closure of the plume equations, Atmos. Environ., 18, 653-662, 1984.

Houghton, H. G. and Cramer, H. E.: A theory of entrainment in convective currents, J. Meteorol., 8, 95-102, 1951.

Hunt, G. R. and Kaye, N. G.: Virtual origin correction for lazy turbulent plumes, J. Fluid. Mech., 435, 377-396, 2001.

Kaminski, E., Tait, S., And Carazzo, G.: Turbulent entraiment in jets with arbitrary buoyancy, J. Fluid. Mech., 526, 361-376, 2005.

Landel, J. R., Caulfield, C. P., and Woods, A.: Meandering due to large eddies and the statistically self-similar dynamics of quasitwo-dimensional jets, J. Fluid Mech., 692, 347-368, 2012.

Lee, J. H. W. and Chu, V. H.: Turbulent jets and plumes: A lagrangian approach, Kluwer Academic Publishers, the Netherlands, 2003.

List, E. J.: Turbulent jets and plumes, Annu. Rev. Fluid Mech. 14, 189-212, 1982.

López, P.: Turbulent mixing by gravitational convection: experimental modelling and application to atmospheric situations, Ph.D. thesis, Complutense University of Madrid, Spain, 2004.

López, P., Cano, J. L., and Redondo, J. M.: An experimental model of mixing processes generated by an array of top-heavy turbulent plumes, Il Nuovo Cimento, 31 C, 679-698, 2008.

Mahjoub, O. B., Redondo, J. M., and Babiano, A.: Structure functions in complex flows, Appl. Sci. Res., 59, 299-313, 1998.

Matulka, A., Redondo, J. M., and Carrillo, A.: Experiments in stratified and rotating decaying 2D flows, Il Nuovo Cimento, $31 \mathrm{C}$, 757-770, 2008.

Morton, B. R.: Forced plumes, J. Fluid Mech., 5, 151-163, 1959.

Morton, B. R.: The choice of conservation equations for plume models, J. Geophys. Res., 30, 7409-7416, 1971.
Morton, B. R., Taylor, G. I., and Turner, J. S.: Turbulent gravitational convection from maintained and instantaneous sources, $\mathrm{P}$. Roy. Soc. Lond. A Mat., 234, 1-23, 1956.

Nielsen, P. V.: Displacement ventilation-theory and design, Ph.D. thesis, Aalborg University, Aalborg, Denmark, 1993.

Redondo, J. M., Fernando, J. H., And Pares, S.: Cloud entrainment by internal or external turbulence, in: Mixing in Geophysical Flows, edited by: Redondo, J. M. and Metais, O., CIMNE, Barcelona, 1995.

Redondo, J. M. and Yagüe, C.: Plume entrainment in stratified flows, in: Recent advances in the fluid mechanics of turbulent jets and plumes, edited by: Davies, P. A. and Valente-Neves, M. J., NATO ASI Series E: Applied Sciences, 255, 209-222, 1994.

Squires, P. and Turner, J. S.: An entraining jet model for cumulonimbus updraughts, Tellus, XIV, 422-434, 1962.

Sreenivas, K. R. and Prasap, A. K.: Vortex dynamics model for entrainment in jets and plumes, Phys. Fluids, 12, 2101, doi:10.1063/1.870455, 2000.

Stommel, H.: Entrainment of air into a cumulus cloud, J. Meteorol., 4, 91-94, 1947.

Tate, P. M.: The rise and dilution of buoyant jets and their behaviour in an internal wave field, Ph.D. thesis, University of New South Wales, Sydney, Australia, 2002.

Turner, J. S.: The starting plume in neutral surroundings, J. Fluid. Mech., 13, 356-368, 1962.

Turner, J. S.: Turbulent entrainment: the development of the entrainment assumption and its application to geophysical flows, J. Fluid Mech., 173, 431-471, 1986.

Vindel, J. M., Yagüe, C., and Redondo, J. M.: Relationship between intermittency and stratfication, Il Nuovo Cimento, $31 \mathrm{C}$, 669-678, 2008.

Wright, S.: Buoyant jets in density-stratified crossflow, J. Hydraul. Eng.-ASCE, 110, 643-656, 1984. 